\title{
Leucocyte elastase activity in meningococcal septicaemia associated coagulopathy
}

\author{
D CANAVAN,* FLORENCE ROBINSON,* P TURKINGTON
}

From the Department of Haematology, Royal Victoria Hospital, Belfast, and the *Infection Unit, Belvoir Park Hospital, Belfast, Northern Ireland

SUMMARY The concentration of the elastase- $\alpha 1$ proteinase inhibitor complex (E- $\alpha 1 \mathrm{PI})$ in a meningococcal infection in an index case with severe changes in haemostasis was measured. The concentration of the E- $\alpha 1$ PI complex was increased throughout the duration of the illness, although concentrations of the blood clotting factors were severely decreased. The release of polymorphonuclear elastase activity may contribute to the depletion in clotting factors.

Disseminated intravascular coagulation is often associated with meningococcal infection. ${ }^{1}$ Recently data have been presented, which suggest that the depletion in blood clotting factors, observed in blood testing, could be the direct result of clotting factor degradation by the action of systemically released polymorphonuclear leucocyte elastase activity. ${ }^{2}$ With the development of a sensitive immunoassay for the elastase- $\alpha 1$ proteinase inhibitor complex (E- $\alpha 1 \mathrm{PI})$, its quantitation in plasma provides an indirect but clear indication of elastase release. ${ }^{3}$ We describe a case of disseminated intravascular coagulation caused by Neisseria meningitidis.

\section{Material and methods}

Clotting factor assays were performed using methods previously described. ${ }^{4}$ Total white cell count, platelet count, one stage prothrombin times (PT), and partial thromboplastin (kaolin) times (PTTK) were estimated using routine tests available in the department.

The estimation of the elastase- $\alpha 1$ proteinase inhibitor complex (E- $\alpha 1$ PI) was performed as previously described, ${ }^{3}$ but with the following changes: standard, test, and control plasmas were diluted $1 / 1000$ in $0.05 \mathrm{M}$ phosphate buffer ( $\mathrm{pH} 7.4)$ containing $0.05 \%$ Tween and $1 \%$ chick serum. The second antibody in the assay was conjugated to peroxidase and used at a dilution of $1 / 800$.

\section{CASE REPORT}

A five year old girl was admitted to hospital with an 18 hour history of malaise, fever, and vomiting followed by the abrupt and rapid appearance of an extensive generalised purpuric and ecchymotic rash.
Culture of cerebrospinal fluid and blood yielded group B meningococci. The child was immediately treated with intravenous penicillin. Ecchymotic spots continued to develop over the next few hours, and at 15 hours (T15) this was followed by the abrupt and rapid appearance of extensive generalised purpuric and ecchymotic rash. The clotting screen repeated at 30 hours (T30) showed a pronounced deterioration in the clotting factor activity and platelet count. The coagulation screen at 34 hours (T34) showed a cons siderable improvement. Thereafter she made a slow steady improvement, her toxaemia settling within 48 hours. About five days after admission all coagulation changes had returned to normal.

\section{Results}

Bacterial analysis identified the organism as Neisseria meningitidis. The results show a time dependent decrease in blood clotting factors (table 1). The concentration of the E- $\alpha 1$ PI complex showed a sustained increase throughout the duration of the illness. One hundred and twenty hours (T120) after admission all coagulation variables, cellular changes, and $E-\alpha 1$ PI complex had returned to normal (table 2).

\section{Discussion}

Meningococcal septicaemia is often associated with disseminated intravascular coagulation, which may lead to severe haemorrhage as a result of thrombocytopaenia and clotting factor depletion. ${ }^{1}$ The clinical course of our patient confirms this association. Although the present study showed clotting factor depletion, no direct evidence of procoagulant activity was found. Recently data have been presented that suggest that the clotting factor depletion observed in 
Table 1 Coagulation changes observed during course of illness

\begin{tabular}{lllllrrr}
\hline Time (hours) & 15 & 30 & 34 & 46 & 96 & 120 & Normal range \\
\hline Prothrombin time & 23 & 26 & 18 & 16 & 14 & 14 & $12-15$ seconds \\
Partial thromboplastin time & 56 & 86 & 60 & 50 & 48 & 42 & $33-55$ seconds \\
Platelet count & 92 & 44 & 140 & 68 & 125 & 150 & $150-145 \times 10^{9} / 1$ \\
\hline
\end{tabular}

Table 2 White cell count and E- $\alpha$ I PI complex concentration observed during course of illness

\begin{tabular}{lccccccc}
\hline Time (hours) & 15 & 30 & 34 & 46 & 96 & 120 & Normal range \\
\hline Total white cell count & $17 \cdot 6$ & $26 \cdot 6$ & $15 \cdot 2$ & $16 \cdot 8$ & $20 \cdot 5$ & $10 \cdot 5$ & $5 \cdot 0-15 \cdot 0 \times 10^{9} / 1$ \\
E $-\alpha 1$ PI activity & 480 & 900 & 680 & 800 & 200 & 100 & $<100 \mu \mathrm{g} / 1$ \\
\hline
\end{tabular}

vitro in septicaemia may be the result of direct proteolysis of the clotting factors by the action of systemically released polymorphonuclear leucocyte elastase activity. ${ }^{2}$ The present case study shows for the first time that a meningococcal infection can be associated with excessive release of elastase activity in vivo. The systemic release of elastase may have contributed to the clotting factor depletion as it has been reported that the proteolysis of blood clotting can occur before the inactivation of elastase is achieved. ${ }^{2}$ The total leucocyte count in the present case study did not show a major increase and therefore was not a useful indicator of the inflammatory response. We showed that the activities of the E- $\alpha 1$ PI complex were considerably increased throughout the infection and that its estimation may be of value in monitoring the inflammatory response. Further studies in this field are required to establish the roles of elastase activity in the aetiology of the clotting factor destruction and in assessing clinical severity.

We thank Professor JM Bridges for his permission to perform the assays in the department of haematology,
Royal Victoria Hospital, Belfast. We thank Chroma Research, Belfast, Northern Ireland for the provision of $\mathrm{E}-\alpha 1 \mathrm{PI}$ control plasmas.

\section{References}

1 Abilgaard CF, Corrigan JJ, Seeler RA, Simone JV, Schulman I. Meningiococcaemia associated with intravascular coagulation. Pediatrics 1967;40:78-84.

2 Egbring R, Schmidt W, Fuschs G, Havemann K. Demonstration of granulocytic proteases in plasma of patients with acute leukaemia and septicaemia with coagulation defects. Blood 1977;49:219-31.

3 Neumann S, Gunzer G, Hennrich N, Lang H. Enzyme immunoassay for human polymorphonuclear elastase complexed with al-proteinase inhibitor. J Clin Chem Clin Biochem 1984;22: 693-7.

4 Thomson JM. Blood coagulation and haemostasis. A practical guide. Edinburgh: Churchill Livingstone, 1980:1-92.

Requests for reprints to: Dr PT Turkington. The University of Kuwait, Faculty of Allied Health Sciences, PO Box 31470, 90805 Sulaibikhat, Kuwait. 